\title{
Automatic Segmentation and Classification of SEM Images of Bacteria Cells
}

\author{
Mangala Shetty, Balasubramani R., Vidya S.M.
}

\begin{abstract}
In the field of microbiology, digital image analysis methods are receiving significant attention to automatically interpret images of bacterial cells. An automatic procedure to extract and classify images of lactic acid bacteria (LAB) is presented in this paper. Edge based watershed method with automatically generated markers were used to retain the image information at fine scales. The experiment was conducted on images containing one type of bacteria. The scanning electron microscopic (SEM) images of lactic acid bacteria (LAB) are used in this experiment. The image analysis and classification technique described in this paper is quick and simple to recognize organisms based on their morphological characteristics. The classification results indicate that routine methods for the detection, enumeration and identification of bacteria can be automated with use of direct microscopic methods.

Keywords: Image classification, bacterial cell analysis, segmentation.
\end{abstract}

\section{INTRODUCTION}

Microscopic image segmentation is another region that is affected by computing methods. In many instances, the medical diagnosis in histology is based on quantitative measures such as calculating the cell strength of a particular cell type or analyzing their shape and measuring their sizes based on the microscopic images. It is highly time-consuming and labour intensive task to classify and identify bacterial cells manually. In order to facilitate the recognition and enumeration of particular bacteria, we have started to explore alternative methods. Such automated methods of bacterial cell image analysis are fast, robust and reliable. So they are very helpful in routine biology.

Lactic acid bacteria are the major groups of microorganisms used in fermentation of food [1]. Size and shapes of bacterial cells are helpful and important morphological characteristics [2],[3] which can be used in the detection of bacteria. Electron microscope has become more and more useful in imaging techniques for microbial study over the past decades [4]. SEM equipment are highly capable of producing high quality images from time lapse experiments [4].The main objective of analyzing an image is to recognize features of an image such as s shape, size, optical density, position and

Revised Manuscript Received on November 15, 2019

Mangala Shetty, Assistant Professor, Department of MCA, NMAM Institute of Technology, Nitte,

Balasubramani R., Professor, EDC Head, Department of Information Science and negineering, NMAM Institute of Technology, Nitte

Vidya S.M., Ph.D. in Biotechnology, Kuvempu University, Karnataka. M.Sc. in Biotechnology, 1999-2001, Mysore University, Karnataka other morphological features. Electron microscopic imaging mechanisms have become very essential and useful to study microbial community. Due to its highly accurate results, easy repetition of tasks and fidelity. The objective of this paper is classifying SEM images of bacterial cells based on cell morphology.

\section{LITRATURE SURVEY}

Recently, a large number of researches have been conducted to extend computer vision and machine learning techniques for the automated analysis of microscopic image of bacteria cells. Among the most recent related works on segmentation of object areas, common approaches are thresholding[1], adaptive thresholding [9], watershed transformation[10], and machine learning techniques[2]-[4],[5] and deep learning methods[6],[7] and active contour model[8]. It is found that manually adapted threshold value does not operate well [1] for heterogeneous background.Several applications in the computer vision community and medical image analysis [11] have adopted the conv -networks. Despite the scope of their uses in various areas, convnets have only recently introduced for biological data analysis, and latest work shows that convnets have important potential to address a biologist's needs in data analysis[13].In the computer vision community, the great success of the Convolutional Neural Network (CNN) in the task of image classification[14], the model of deep learning has been widely accepted. In recognizing objects well-trained deep conv-net architecture shows excellent performance compare to humans. Support Vector Machine (SVM) [14] is a popular non-parametric technique for binary classification.

\section{PROPOSED METHODOLOGY}

The Proposed method is involves following three approaches as depicted in Fig. 1.

\section{A. Noise Removal}

Noise is undesirable data contaminating an image. Noise presents images during capturing phase and also because of the imaging device's optical properties. Removing noise while preserving image quality is the very first step in our methodology. We used morphological operations to remove the noise with distinct structuring elements. Dilation, erosion, opening, and closing are the various operations conducted. We have integrated opening and closing activities with various structuring components to obtain

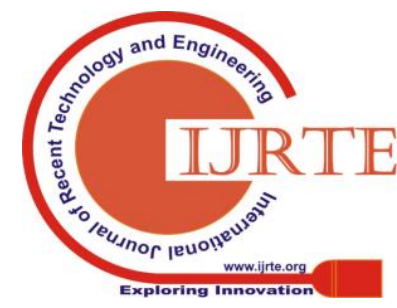




\section{Automatic Segmentation and Classification of SEM Images of Bacteria Cells}

more sophisticated results.

\section{B. Image Analysis and Classification}

The objects from bacterial cell image are separated from the background in order to isolate relevant objects. The low contrast presents an image segmentation challenge. The segmentation shall be carried out as described i.e. segmenting the initial gray scale image using the proposed edge based watershed method with automatically generated markers technique. Marker based morphological segmentation of the watershed imitates the landscape's natural phenomena. The mountains are like the ridge lines and valleys are the catchment basins in the landscape. This method involves the use of foreground and background markers for computing the object of choice and background pixels. The pixel values of high intensity correspond to ridge lines and pixels with lower intensity correspond to valley points in the image. A valley is a elevation area surrounded by lines of ridge [17][18]. To decrease the issue of over segmentation, we used the edge-based gradient magnitude image and applied watershed transformation technique.

This technique includes the application of background and foreground markers for selecting foreground and background pixels. Literature survey gives information about various marker calculation techniques such as connectivity and clustering based on image features, gray-level values, and using neural networks, but in this paper, marker-controlled morphological procedures are used. The methods reconstruction based closing and opening are used to calculate foreground markers. These methods yield is the flat maxima inside each object in the image. Soon after completing this process obtain regional maxima points for every foreground object. This technique is very efficient because it removes only the tiny defects without influencing the object's morphology. Superimposing images of foreground markers on the initial image to view the outcome. The background markers were acquired by first taking the internal marker complement followed by the morphological erosion. These external markers split the image efficiently into distinct areas, each region having a single internal marker and context. The watershed transition is calculated using the morphologic techniques computed with both foreground and background markers. Fig. 2, shows sample images used in the process.

\section{Algorithm}

Step 1: Modify the input image to grayscale image.

Step 2: Use edge detection function to develop gradient images.

Step 3: Select the foreground areas of the image using morphological reconstruction.

Step 4: In order to get the best foreground markers, calculate regional maxima and minima.

Step 5: Superimpose the initial image with the marker.

Step 6: Use edge reconstruction to clean the marker edges.

Step 7: Calculate markers for the background.

Step 8: Modify the gradient image with foreground and background markers through morphological reconstruction.

Step 9: Watershed transformation application to altered gradient image provides final segmented images of required objects.

Step 10: Perform segmented image labeling after removing boundary touching cells.

Step 11: Calculate geometric shape characteristics (i.e. length-width ratio, tortuosity, compactness, circularity, and eccentricity) for each cell type.

Step 12: For all the training images, repeat steps 1 to 6 .

Step 13: Calculate standard deviation, mean of the features values for every feature extracted in the step 11.

Step 14: Apply bacterial cell classification operations.

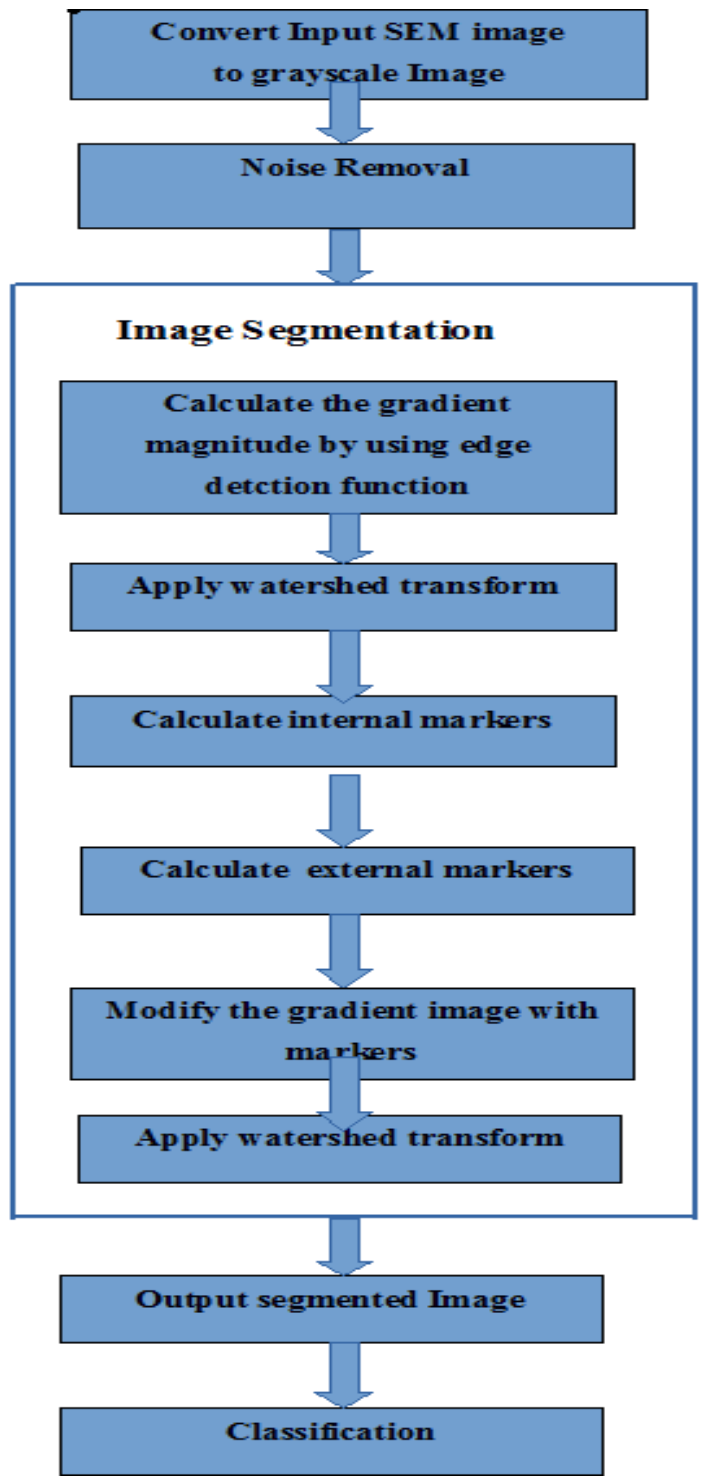

Fig: 1. Proposed Approach

The above classification algorithm classification is done by different classifiers such as SVM, KNN and CNN. Accuracy of classification is compared. 

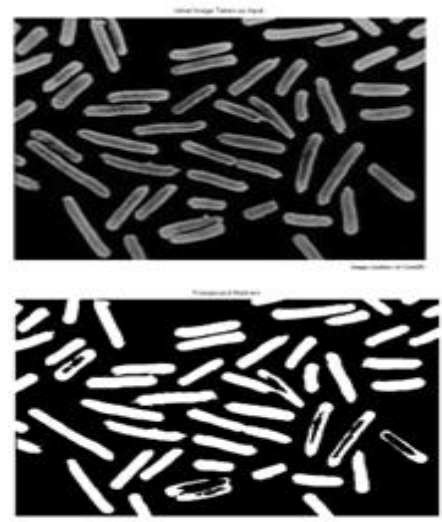
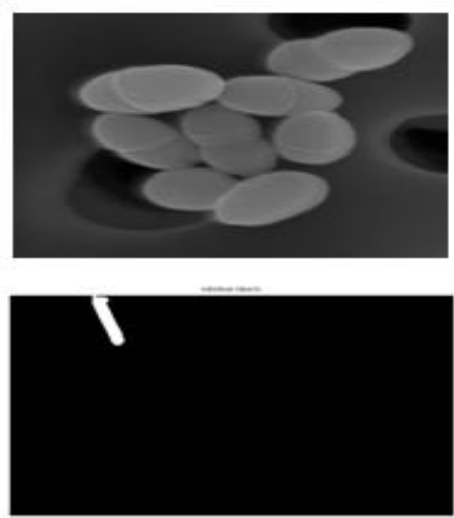
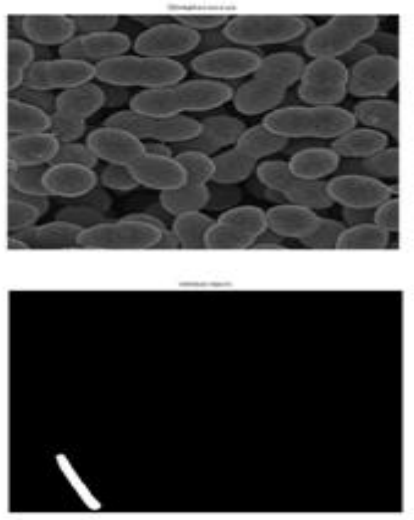

Fig. 2. First row: Sample input images used for classification process Second row: Output Images.

As the class is determined on the basis of geometrical characteristics, primary morphological characteristics are extracted. The aim of the SEM image analysis of lactic acid bacteria is to define the type of bacteria whether they are lactobacillus or lactococcus or or staphylococci on the basis of morphological shape features. Among the geometric characteristics used by different writers in the literature[4],[7],[27],[28], five geometric characteristics are noted, namely eccentricity circularity, tortuosity, compactness, and length-width ratio, which gives good classification outcomes. In this paper the following five characteristics are applied on SEM images of bacterial cells. Circularity $\left(\mathrm{x}_{1}\right):(4 *$ pi $*$ Area $) /$ perimeter $^{2}$

Compactness $\left(\mathrm{x}_{2}\right)$ : Compactness is defined as the ratio of the area of an object to the area of a circle with the same perimeter, perimeter ${ }^{2} /\left(4^{*} \mathrm{pi}^{*}\right.$ Area $)$.

Eccentricity $\left(\mathrm{x}_{3}\right)$ : It is the length ratio of the shape's highest chord to the longest chord perpendicular to it. i.e. Length major_axis /Length minor_axis .

$\operatorname{Tortuosity}\left(\mathrm{x}_{4}\right)$ : major axis/perimeter.

Length-width ratio( $\left.\mathrm{x}_{5}\right)$ : major axis/minor axis.

\section{EXPERIMENTAL RESULTS AND DISCUSSIONS}

In this experiment input for image analysis and classification three types of lactic acid bacterial cells are considered. The images divided into two groups testing group and training group. In training phase 90 images were used and about 60 images testing set were taken to assess the classification accuracy.

With CNN classifier, the classification experiment is conducted and obtained 99 percent of accuracy. Table 1 summarizes the outcomes and Fig. 3 shows the chart representation of the same. The $\mathrm{CNN}$ classifier provides most precise class predictions for lactobacillus whereas lowest accuracy predicted by SVM for lactococcus.

In Table 2, mean as well as standard deviation of the geometric features obtained for lactic acid bacteria cells are given.

The gained precision of classification raises the question of possible sources of mistake. Obviously, since in the segmentation stage there clinical observation on detecting overlapping bacterial cells are executed. So the information is bound to include a substantial quantity of overlapping cells with morphological degeneration.

The accuracy of classification confirms that the characteristics of morphology are informative for the suggested classification issue.

Table-I: Classification Accuraccy

\begin{tabular}{|c|c|c|c|c|}
\hline \multicolumn{4}{|c|}{ Classification accuracy (\%) } \\
\hline Cell Types & \multicolumn{3}{|l|}{$\begin{array}{l}\text { SVM } \\
\text { Classifier }\end{array}$} & \multicolumn{2}{|c|}{ K-NN classifier } & $\begin{array}{c}\text { Neural } \\
\text { Network } \\
\text { classifier }\end{array}$ \\
\cline { 3 - 4 } & $\mathrm{k}=1$ & $\mathrm{k}=3$ & $99 \%$ \\
\hline Lactobacillus & $80 \%$ & $87 \%$ & $89 \%$ & $98 \%$ \\
\hline Streptococcus & $79 \%$ & $86 \%$ & $88 \%$ & $98.6 \%$ \\
\hline Lactococcus & $75 \%$ & $82 \%$ & $85 \%$ & \\
\hline
\end{tabular}

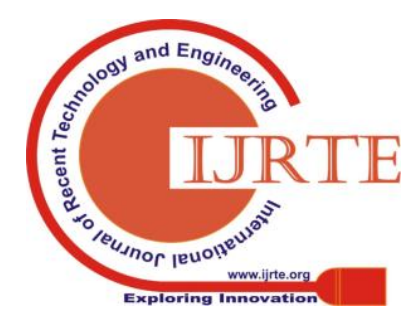


Table-II: Geometric Parameters of the cells

\begin{tabular}{|c|c|c|c|c|c|c|c|c|c|c|}
\hline Cell Type & \multicolumn{2}{|c|}{ Diameter } & \multicolumn{2}{|c|}{ Tottuosity } & \multicolumn{2}{c|}{ Circularity } & \multicolumn{2}{c|}{ Compactness } & \multicolumn{2}{c|}{$\begin{array}{c}\text { Length-Width } \\
\text { Ratio }\end{array}$} \\
\hline \multirow{2}{*}{ Lactobacillus } & \begin{tabular}{c} 
Mean \\
\cline { 2 - 11 }
\end{tabular} & $\begin{array}{c}\text { Standard } \\
\text { Deviation }\end{array}$ & Mean & $\begin{array}{c}\text { Standard } \\
\text { Deviation }\end{array}$ & Mean & $\begin{array}{c}\text { Standard } \\
\text { Deviation }\end{array}$ & Mean & $\begin{array}{c}\text { Standard } \\
\text { Deviation }\end{array}$ & $\begin{array}{c}\text { Mean } \\
\text { Standard } \\
\text { Deviation }\end{array}$ \\
\hline Streptococcus & 0.9651 & 0.0273 & 0.3952 & 0.0333 & 0.3142 & 0.2102 & 3.7141 & 0.8241 & 4.5236 & 1.0210 \\
\hline Lactococcus & 1.3306 & 0.0257 & 0.3439 & 0.0314 & 0.5987 & 0.4213 & 1.6702 & 0.3421 & 1.4016 & 0.1461 \\
\hline
\end{tabular}

\section{CONCLUSION}

We have suggested an automated model in this paper for lactic acid bacteria cell image analysis by proposing a method for segmenting the images of bacterial cell and to extract geometric features from the cells. The described technique is less expensive and produces a significant set of classification rates. It has produced results in the range of $81 \%$ to $99 \%$ with SVM classification having reached $75 \%$ to $80 \%$ and $98 \%$ to $99 \%$ with CNN classifier. The suggested technique is computerized less costly and results similar levels in the range.

Better techniques and function sets which can be adopted in our future work could be further enhanced. In addition, the results of the experiment were very well correlated with the population of the reference. The findings in this paper motivate the further investigation on bacterial cell image analysis. The method can discover its use in the medical sector in the tentative identification of cells in clinical specimens and in the environmental sector in order to recognize the existence of helpful crops in addition to use in systematic study labs. It can also be used to rapidly determine the existence of possible contaminants in food industries.

\section{ACKNOWLEDGMENT}

Authors are thankful to Dr. Dennis Kunkel, Formerly president at Dennis Kunkel Microscopy Inc. for providing the SEM images.

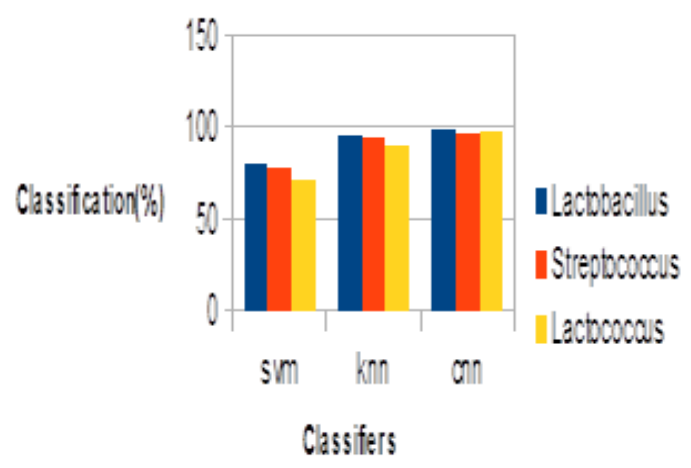

Fig.3 Classification Accuracy chart

\section{REFERENCES}

1. N. Otsu, "A threshold selection method from gray-level histograms," IEEE Trans. Syst., Man, Cybern., vol. 9, no. 1, pp. 62-66, Jan. 1979.

2. L. Gorelick et al., "Prostate histopathology learning tissue component histograms for cancer detection and classification," IEEE Trans. Med. Imaging, vol. 32, no. 10, pp. 1804-1818, Oct, 2013.

3. Y. Y. Xu, F. Yang, Y. Zhang, and H. B. Shen, "Bioimaging-based detection of mislocalized proteins in human cancers by semi-supervised learning," Bioinformatics, vol. 31, no. 7, pp. 1111-1119, 2015.

4. Y. Xu, J. Y. Zhu, E. I. Chang, M. Lai, and Z. W. Tu, "Weakly supervised histopathology cancer image segmentation and classification," Med. Image Anal., vol. 18, no. 3, pp. 591-604, 2014.

5. J. Chang et al., "Automated tuberculosis diagnosis using fluorescence images from a mobile microscope," in Proc. 15th Int. Conf. Med. Image Comput. Comput.-Assisted Intervention, 2012, pp. 345-352.

6. G. E. Hinton, "Learning multiple layers of representation," Trends Cogn. Sci., vol. 11, no. 10, pp. 428-434, 2007.

7. Y. Y. Song, L. Zhang, S. P. Chen, D. Ni, B. Y. Lei, and T. F. Wang, "Accurate segmentation of cervical cytoplasm and nuclei based on on multi-scale convolutional network and graph partitioning," IEEE Trans. Biomed. Eng., vol. 62, no. 10, pp. 2421-2433, Oct. 2015. DOI 10.1109/TBME.2015.2430895.

8. T. F. Chan and A. V. Luminita, "Active contours without edges," IEEE Trans. Image Process., vol. 10, no. 2, pp. 266-277, Feb. 2001.

9. O. D. Trier and A. K. Jain, "Goal-directed evaluation of binarization methods," IEEE Trans. Pattern Anal. Mach. Intell., vol. 17, no. 12, pp. 1191 -1201, Dec. 1995.

10. L. Vincent and P. Soille, "Watersheds in digital spaces: An efficient algorithm based on immersion similations," IEEE Trans. Pattern Anal. Mach. Intell., vol. 13, no. 6, pp. 583-598, Jun. 1991. Noh, H., Hong, S. \& Han, B., 2015. Learning deconvolution network for semantic segmentation.

11. Tajbakhsh, N. et al., 2016 "Convolutional Neural Networks for Medical Image Analysis: Full Training or Fine Tuning" IEEE Transactions on Medical Imaging, May, 35(5), pp. 1299-1312.

12. Van Valen, D. A. et al., 2016. "Deep learning automates the quantitative analysis of individual cells in live-cell imaging experiments." PLoS Comput Biol, November, 12(11), pp. 1-24.

13. Alex Krizhevsky, Ilya Sutskever, and Geoffrey E. Hinton. "Imagenet classication with deep convolutional neural networks" Neural Information Processing Systems, 2012.

14. Cortes, C., Vapnik, V., "Support-vector networks", Machine Learning 20(3) (1995) 273-297.

15. Yosinski J, Clune J, Bengio Y, Lipson H.” How transferable are features in deep neural networks" Proceedings of the 27th International Conference on Neural Information Processing Systems-Volume 2; Montreal, Canada. 2969197: MIT Press; 2014. p. 3320-8.

16. D. H. Wolpert, "The lack of a priori distinctions between learning algorithms," Neural Comput. 8(7), 1341-1390 (1996).

17. Ng, H.P., Ong, S.H., Foong, K.W.C., Goh, P.S., Nowinski, W.L. "Medical image segmentation using clustering of K-means and improved watershed algorithms" 2006 IEEE Southwest Image Analysis and

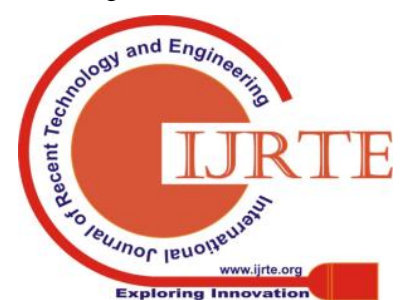


Interpretation Symposium, pp. 61-65 (2006).

18. Gonzalez, R.C., Woods, R.E. processing of digital images. Hall of the Prentice (2002)

19. K. Rodenacker, together with E. Bengtsson. "A function set on digitized microscopic images for cytometry" Anal Cell Pathol, 25(1):1-36, 2003

\section{AUTHORS PROFILE}

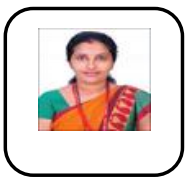

Ms. Mangala A.G Assistant Professor, Department of MCA, NMAM Institute of Technology, Nitte, MCA,(Ph.D). Qualified MCA in 2007 from Mangalore University Mangalore. Completed Bachelor in Science in 2002 from Dr. Nitte Shankara Adyanthaya Memorial FGC, Nitte. Publications: Mangala A.G. and Balasubramani R., "Efficient 2-D Structuring Element for Noise Removal of Grayscale Images Using Morphological Operators", IJCA (International Journal of Computer Applications), ISBN : 973-93-80893-39-6, DOI: 10.5120/ijca2016910188, pp 24-28, Volume 143, Number 6, 2016, Presented paper on "Lactobacillus Cell Segmentation Based on Marker Controlled Watershed Method" at $3^{\text {rd }}$ IEEE International Conference on "Elrctrical, Electronics, Communication, Computer Technologies and Optimization Techniques" (ICEECCOT-2018).She has Guided more than fifty student projects. Organized Cryptogrphy in Network Security Workshop at NMAMIT Nitte in the year 2017. Areas of interest includes Bioinformatics, cloud computing, biological image processing.

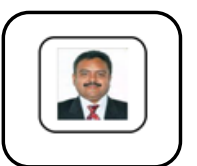

Dr. Balasubramani R, Professor, EDC Head, Department of Information Science and negineering, NMAM Institute of Technology, Nitte, Ph.D. in Content Based Imaged Retrieval for Stock Photography using Semantic and Visual Descriptors from Vinayaka Missions Research Foundation (Deemed-to-be-University) Salem in 2011, M.Tech. in Information Technology from Allahabad Agricultural Institute (Deemed-to-be-University), Allahabad in 2005. Qualified in the AICTE Deemed to be University Examination 2018. B.E. in Electronics and Communication Engineering from Madurai Kamaraj University, Madurai in 1990, Publications: "Attainment of Programme Outcomes through Course Outcomes in Outcome Based Education: A Case Study", Journal of Engineering Education Transformations (JEET) | Online ISSN: 2394-1707, Print ISSN: $2349-2473$ | DOI: $10.16920 /$ jeet/2017/v31i2/119555 |pp 26-30 | Volume: 31 | No: 02 | October 2017.personal profile which contains their education details, their publications, research work, membership, achievements, with photo that will be maximum 200-400 words Balasubramani R., Devidas, "Enhancing Employability Skills through Infosys Campus Connect Programme: A Case Study", JEET (Journal of Engineering Education Transformations), Online ISSN: 2394-1707, Print ISSN: $2349-2473$, DOI: $10.16920 /$ jeet/2015/v29i1/77113, pp 60-66, Volume 29, Issue 1, July 2015. Balasubramani R, Manasa I P, "Multi-Level Network Scheduling Scheme for Wireless Sensor Network", IJSR-International Journal of Scientific Research, ISSN No. 2277 - 8179, pp 282-285, Volume: 3 , Issue: 5, May 2014. Balasubramani R, Kannan V, "Semantic Annotation of Stock Photography for CBIR using MPEG-7 standards", Global Journal of Computer Science \& Technology 2009(GJCST 2009), Publisher: Global Journals Inc.,(USA), ISSN: 0975-4172(Online), 0975-4350(Print), pp 7-11, Vol. 9 , Issue 5 , September 2009. Balasubramani R, Kannan V, "Semantic Search and Retrieval of Stock Photography based on MPEG-7 Descriptors", Global Journal of Computer Science \& Technology 2009(GJCST 2009), Publisher: Global Journals Inc.,(USA), ISSN: 0975-4172(Online), 0975-4350(Print), pp 146-156, Vol. 9 , Issue 5 , September 2009. Balasubramani R, Kannan V, "Efficient use of MPEG-7 Color Layout and Edge Histogram Descriptors in CBIR Systems", Global Journal of Computer Science \& Technology 2009(GJCST 2009), Publisher: Global Journals Inc.,(USA), ISSN: 0975-4172(Online), 0975-4350(Print), pp 157-163 , Vol. 9 , Issue 5 , September 2009.

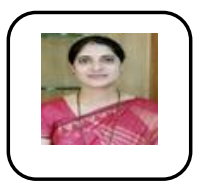

Dr. Vidya S. M., Ph.D. in Biotechnology, 2002-2006. Kuvempu University, Karnataka. M.Sc. in Biotechnology, 1999-2001, Mysore University, Karnataka. Life Member of 'The Indian Science Congress Association'. Life Member of 'The Society of Biological Chemists', India. Life Member of 'Society For Free Radical Research', India. Received award for research publications by Vision Group of Science and Technology, Government of Karnataka during the year 2017-18. Applied patent on Super-sensitive and stable gold nanoparticles- 6186/CHE/2014 filed on 08/12/2014. Nitte University sponsorship for research program at Penn State University, Harrisburg, 2013. Indian Drug Manufacturers' Association Best Research Award 2004. Sponsored Research includes Investigation on the role of probiotic exopolysaccharides in the prevention of radiation induced injury, 2014-17 DAE/BRNS, INR 22.13 Lakhs.Investigation on the role of L.rhamnosus exopolysaccharide on biofilm formation and its radioprotective application, 2016-17 TEQIP Phase II - Res Grant, NMAMIT, INR 1 Lakh. Production and distribution of tissue culture plantlets of banana var, 'Grand Naine' for the economic empowerment of the rural marginal SC/ST farmers and weaker sections of Udupi District, Karnataka, 2012-15 DBT, INR 23.00 Lakhs. In vivo studies on comparative and synergistic radioprotective effect of stem bark extracts of Ficus racemosa, Madhuca indica and Pterocarpus marsupium based on ethnomedicinal knowledge to cancer treatment, 2010-13 DAE/BRNS, INR 16.16 Lakhs. 